\title{
On the Dual Decomposition of Linear Quadratic Optimal Control Problems for Vehicular Formations
}

\author{
Makan Fardad, Fu Lin, and Mihailo R. Jovanović
}

\begin{abstract}
We use the dual decomposition method along with the dual subgradient algorithm to decouple the linear quadratic optimal control problem for a system of single-integrator vehicles. This produces the optimal control law in a localized manner, in the sense that vehicles can iteratively compute their primal and dual variables by only communicating with their immediate neighbors. In particular, we demonstrate that each vehicle only needs to receive the primal variable of the vehicle ahead and the dual variable of the vehicle behind. We then assume a structured feedback gain relationship between the state and actuation signals, and reformulate the optimization problem to find the optimal feedback gains. We develop an algorithm whereby vehicles can compute structured feedback gains in a localized manner. Convergence properties of the latter algorithm are improved by employing a relaxed version of the augmented Lagrangian method, and numerical examples are provided to demonstrate the utility of our results.
\end{abstract}

Index Terms - Distributed optimization, dual decomposition, subgradient algorithm, localized cooperative control, multivehicle systems.

\section{INTRODUCTION}

It is well-known that the feedback gain obtained from solving an LQR problem for a system with banded statespace matrices is, in general, a full matrix. This means that even if the subsystems interact locally, the optimal controller will need global information in order to produce the feedback signal. This is an undesirable scenario for large networks of dynamical systems, owing to its excessive communication requirements. A preferred alternative is to have control signals computed using only local communication among neighboring subsystems. A strong motivation for use of such inherently local controllers comes from [1], where it was demonstrated that the dependence of a controller on information coming from other parts of the system decays exponentially as one moves away from that controller

Over the years a body of literature has been developed that addresses the problem of distributed control of interconnected systems [1]-[13]. The present work is motivated by [14], [15]. In [14] an iterative algorithm is proposed by which distributed controllers determine the optimal localized feedback gains using gradient methods. In [15] the dual decomposition method is used to decouple and analyze large-scale optimal control problems. Other related works that employ dual decomposition techniques

Financial support from the National Science Foundation under awards CMMI-0927509 and CMMI-0927720 and under CAREER award CMMI0644793 is gratefully acknowledged.

M. Fardad is with the Department of Electrical Engineering and Computer Science, Syracuse University, NY 13244. F. Lin and M. R. Jovanović are with the Department of Electrical and Computer Engineering, University of Minnesota, Minneapolis, MN 55455. E-mails: makan@syr.edu, fu@umn.edu, mihailo@umn.edu. for distributed control are [16], [17].

In this paper we consider the linear quadratic control of a single-integrator system of vehicles. Via a change of variables we convert this problem to the so-called separable form, in which independent optimization problems are coupled only through their constraint equations. Using Lagrange multipliers, the problem fully decouples into individual optimization subproblems for each of the vehicles. Primal and dual variables are then updated locally and iteratively at every vehicle through a dual subgradient algorithm. This procedure is localized in the sense that at every iteration vehicles need to pass their primal and dual variables only to their immediate neighbors in order to compute their actuation signals. Furthermore, we employ the dual subgradient algorithm to compute localized structured feedback gains for the system of vehicles.

The rest of the paper is organized as follows. In Section II we formulate the control problem over a finite time horizon for a system of single-integrator vehicles. In Section III we introduce a change of variables which transforms the problem to separable form. In Section IV we apply the dual decomposition method and show that the solution of the optimal control problem (i.e., the profiles of the state and actuation signals over the entire time horizon) can be determined in a localized fashion. In Section V we assume a structured feedback gain relationship between state and actuation signals, and reformulate the optimization problem to find the optimal feedback gains. We show that the necessary conditions for optimality of this nonconvex problem come in the form of fifth-order polynomial equations (in the unknown feedback gains) whose solutions identify all the extremum points of the Lagrangian. In Section VI we use a relaxed version of the augmented Lagrangian method to convexify the structured feedback gain design problem, and in Section VII we provide numerical examples to demonstrate the utility of our results.

\section{Problem Formulation}

We consider the discrete-time optimal control problem for a first-order model of a system of $N$ vehicles,

$$
\begin{array}{cc}
\text { minimize } & \frac{1}{2} \sum_{k=0}^{K} x_{1}^{2}+\left(x_{2}-x_{1}\right)^{2}+\cdots+\left(x_{N}-x_{N-1}\right)^{2} \\
+ & \frac{1}{2} \sum_{k=0}^{K-1} u_{1}^{2}+u_{2}^{2}+\cdots+u_{N}^{2} \\
\text { subject to } \quad x_{n}(k+1) & =a x_{n}(k)+u_{n}(k), \\
n & =1, \ldots, N, \\
k & =0, \ldots, K-1 .
\end{array}
$$


This is an LQR problem with $A=a I, B=R=I$, and

$$
Q \sim\left[\begin{array}{rrr}
1-1 & & \\
1 & -1 & \\
& 1 & -1 \\
& & 1
\end{array}\right]\left[\begin{array}{rrrr}
1 & & & \\
-1 & 1 & & \\
& -1 & 1 & \\
& & -1 & 1
\end{array}\right]=\left[\begin{array}{rrrr}
2 & -1 & & \\
-1 & 2 & -1 & \\
-1 & 2 & -1 \\
& -1 & 1
\end{array}\right] \text {. }
$$

Since $Q$ is not a diagonal matrix, it can be shown that the solution of the algebraic Riccati equation (and thus the optimal feedback gain) will be a full matrix. Such a solution requires communication between all vehicles. In what follows, we will reformulate the problem with the aim of finding an algorithm through which the control signal can be obtained in a 'localized' fashion.

We next demonstrate a method based on dual decomposition in which only neighboring vehicles need to communicate to each other in order to iteratively reach the optimal control signal. We observe that every vehicle transmits its primal variables to the vehicle immediately behind, and transmits its dual variable to the vehicle immediately ahead. In this method, once the iterations are complete, each vehicle will have computed its entire optimal actuation profile from $k=0$ to $k=K-1$.

\section{Reformulation In SEPARABle Form}

There are multiple ways of reformulating problem (LQR) so that it can be decoupled using dual methods. One way of achieving this is to obtain an unconstrained version of (LQR) by eliminating the state-trajectory variables using the state equation constraints. The resulting unconstrained problem can then be split into subproblems by introducing auxiliary variables and applying the standard dual decomposition technique [18]. The minimization of the Lagrangian for each subproblem in this approach, however, requires the inversion of a matrix whose size depends on the length of the time-horizon [16].

To elaborate, (LQR) can be rewritten as

$$
\begin{array}{ll}
\operatorname{minimize} & X_{1}^{T} X_{1}+U_{1}^{T} U_{1} \\
& +\sum_{n=2}^{N}\left(X_{n}-X_{n-1}\right)^{T}\left(X_{n}-X_{n-1}\right)+U_{n}^{T} U_{n}
\end{array}
$$

subject to $X_{n}=G U_{n}+F x_{n}(0), \quad n=1, \ldots, N$,

where

$$
X_{n}=\left[x_{n}(1) \cdots x_{n}(K)\right]^{T}, \quad U_{n}=\left[u_{n}(0) \cdots u_{n}(K-1)\right]^{T} \text {, }
$$

and the $K \times K$ system-matrix $G$ is Toeplitz lower triangular with 1 on its main diagonal and $a^{j}$ on its $j$ th lower subdiagonal, and the $K \times 1$ input-matrix $F$ has its $j$ th element equal to $a^{j}$. Substituting the equations for $X_{n}$ into the objective function and rearranging terms gives

$$
\operatorname{minimize} \quad J_{1}\left(V_{1}\right)+J_{2}\left(V_{2}, V_{1}\right)+\cdots+J_{N}\left(V_{N}, V_{N-1}\right)
$$

where $V_{n}$ is a vector composed of $U_{n}$ and $x_{n}(0)$, and $J_{n}$ is an appropriately defined quadratic form. Decoupling at the level of the objective function can be achieved by rewriting the problem as

$$
\begin{array}{ll}
\operatorname{minimize} & J_{1}\left(V_{1}\right)+J_{2}\left(V_{2}, W_{2}\right)+\cdots+J_{N}\left(V_{N}, W_{N}\right) \\
\text { subject to } & W_{2}=V_{1}, \cdots, W_{N}=V_{N-1}
\end{array}
$$

Reference [18] describes how the dual decomposition algorithm can now be applied to this problem. In particular, the problem of Lagrangian minimization decouples into the minimization of smaller subproblems corresponding to each of the vehicles [18].

We note that in this formulation $J_{n}$ is a quadratic function and hence the resulting Lagrangian is also quadratic. This means that minimizing each of the resulting subproblems amounts to solving a linear equation whose size grows with $K$. We next formulate (LQR) differently, such that Lagrangian minimizations can be obtained without the need for solving linear systems of equations. Furthermore, it turns out that this new formulation is more appropriate for the design of structured feedback gains in Section V.

We now present a different reformulation of (LQR). Reference [19, Sec. 14.4] describes the class of 'separable problems' that are amenable to the application of dual methods. These are problems of the form

$$
\begin{array}{ll}
\text { minimize } & \sum_{n=1}^{q} J_{n}\left(Z_{n}\right) \\
\text { subject to } & \sum_{n=1}^{q} H_{n}\left(Z_{n}\right)=0 .
\end{array}
$$

where $Z=\left[Z_{1}^{T} \cdots Z_{q}^{T}\right]^{T}$ is the vector of optimization variables.

To put problem (LQR) in separable form, we perform the following change of variables

$$
\begin{aligned}
\psi_{1}(k) & :=x_{1}(k), \\
\psi_{n}(k) & :=x_{n}(k)-x_{n-1}(k), \quad n=2, \ldots, N .
\end{aligned}
$$

The optimal control problem now becomes

$$
\begin{aligned}
& \begin{array}{r}
\operatorname{minimize} \\
\frac{1}{2} \sum_{k=0}^{K-1}\left(\psi_{1}^{2}+u_{1}^{2}\right)+\cdots+\left(\psi_{N}^{2}+u_{N}^{2}\right) \\
+\frac{1}{2}\left[\psi_{1}^{2}(K)+\cdots+\psi_{N}^{2}(K)\right]
\end{array} \\
& \text { subject to } \psi_{1}(k+1)=a \psi_{1}(k)+u_{1}(k) \text {, } \\
& \psi_{n}(k+1)=a \psi_{n}(k)+u_{n}(k)-u_{n-1}(k), \\
& n=2, \ldots, N \text {, } \\
& k=0, \ldots, K-1 \text {. }
\end{aligned}
$$

To simplify further, for the $n$th vehicle we define the vector of unknowns $Z_{n}$ as the vector that contains both the entire state trajectory $\Psi_{n}$ and the entire control profile $U_{n}$,

$$
Z_{n}=\left[\begin{array}{c}
\Psi_{n} \\
U_{n}
\end{array}\right], \Psi_{n}=\left[\begin{array}{c}
\psi_{n}(1) \\
\vdots \\
\psi_{n}(K)
\end{array}\right], U_{n}=\left[\begin{array}{c}
u_{n}(0) \\
\vdots \\
u_{n}(K-1)
\end{array}\right] .
$$

The optimization problem can be written as

$$
\begin{array}{ll}
\text { minimize } & \frac{1}{2} \operatorname{tr}\left(Z_{1}^{T} Z_{1}\right)+\cdots+\frac{1}{2} \operatorname{tr}\left(Z_{N}^{T} Z_{N}\right) \\
\text { subject to } & C_{1} Z_{1}+\cdots+C_{N} Z_{N}+c=0
\end{array}
$$

where

$$
C_{1}=\left[\begin{array}{cc}
\Delta & -I \\
0 & I \\
\vdots & \vdots \\
0 & 0 \\
0 & 0
\end{array}\right], \cdots, C_{N-1}=\left[\begin{array}{cc}
0 & 0 \\
0 & 0 \\
\vdots & \vdots \\
\Delta & -I \\
0 & I
\end{array}\right], C_{N}=\left[\begin{array}{cc}
0 & 0 \\
0 & 0 \\
\vdots & \vdots \\
0 & 0 \\
\Delta & -I
\end{array}\right],
$$


$c=-\left[\begin{array}{c}a \Psi_{1}^{0} \\ a \Psi_{2}^{0} \\ \vdots \\ a \Psi_{N}^{0}\end{array}\right], \Delta=\left[\begin{array}{cccc}1 & & & \\ -a & 1 & & \\ & \ddots & \ddots & \\ & & -a & 1\end{array}\right], \Psi_{n}^{0}=\left[\begin{array}{c}\psi_{n}(0) \\ 0 \\ \vdots \\ 0\end{array}\right]$

Since the optimization problem (H2') has a quadratic objective and a linear constraint, it is convex. More importantly, problem (H2') is now in separable form. We exploit this special structure with the use of dual methods in the next section.

\section{Dual Decomposition And Optimal Control}

We form the Lagrangian

$$
\begin{aligned}
L= & \frac{1}{2} \operatorname{tr}\left(Z_{1}^{T} Z_{1}\right)+\cdots+\frac{1}{2} \operatorname{tr}\left(Z_{N}^{T} Z_{N}\right) \\
& +\operatorname{tr}\left(\Lambda^{T}\left[C_{1} Z_{1}+\cdots+C_{N} Z_{N}+c\right]\right) .
\end{aligned}
$$

The KKT conditions for optimality are ${ }^{1}$

$$
\begin{gathered}
\frac{\partial L}{\partial Z_{n}}=0 \Longrightarrow Z_{n}=-C_{n}^{T} \Lambda, \quad n=1, \ldots, N, \\
\frac{\partial L}{\partial \Lambda}=0 \Longrightarrow C_{1} Z_{1}+\cdots+C_{N} Z_{N}+c=0 .
\end{gathered}
$$

Remark 1: In what follows, we will not attempt to solve directly the system of equations above. The reason is that the solver would have to have access to the states of all vehicles. To see this, note that from substituting (KKT1) into (KKT2) we obtain $\Lambda=\left(C_{1} C_{1}^{T}+\cdots+C_{N} C_{N}^{T}\right)^{-1} c$ and thus $Z_{n}=-C_{n}^{T}\left(C_{1} C_{1}^{T}+\cdots+C_{N} C_{N}^{T}\right)^{-1} c$. Therefore, for systems with a large number of vehicles (i) the solver would have to invert a large matrix, the inverse of which is a full matrix; and (ii) the solver would have to have access to the original states of all vehicles in order to form the vector $c$. Therefore $Z_{n}$ can only be computed in a centralized manner, which is an undesired scenario.

Instead of directly solving the KKT conditions, we use the following iterative dual decomposition algorithm [17], [20] to solve the above necessary conditions for optimality.

\section{Dual subgradient algorithm. repeat}

1. For given $\Lambda$, minimize Lagrangian in $Z_{n}$ to obtain $Z_{n}=-C_{n}^{T} \Lambda, n=1, \ldots, N$.

2. Update dual variables $\Lambda^{+}=\Lambda+s\left(C_{1} Z_{1}+\cdots+\right.$ $\left.C_{N} Z_{N}+c\right)$ for given step size $s$.

until stopping criterion $\left\|C_{1} Z_{1}+\cdots+C_{N} Z_{N}+c\right\|<\varepsilon$ is satisfied.

We now demonstrate that the above dual decomposition algorithm works in a localized fashion, which makes it desirable from a distributed control point of view. Let us partition $\Lambda$ as

$$
\Lambda=\left[\begin{array}{c}
\Lambda_{1} \\
\Lambda_{2} \\
\vdots \\
\Lambda_{N}
\end{array}\right]
$$

\footnotetext{
${ }^{1}$ Since the problem is convex, the KKT conditions are both necessary and sufficient for optimality.
}

Then, in Step 1 of the algorithm we compute for $n=$ $1, \ldots, N$,

$$
\left[\begin{array}{c}
\Psi_{n} \\
U_{n}
\end{array}\right]=Z_{n}=-C_{n}^{T} \Lambda=\left[\begin{array}{c}
-\Delta^{T} \Lambda_{n} \\
\Lambda_{n}-\Lambda_{n+1}
\end{array}\right],
$$

with $\Lambda_{N+1}=0$. It is clear that every vehicle can carry out Step 1 of the algorithm locally, by communicating its dual variable with its immediate neighbor; vehicle 1 only needs to know $\Lambda_{2}$, vehicle 2 only needs to know $\Lambda_{3}$, and so on. Furthermore, Step 2 of the algorithm gives

$$
\left[\begin{array}{c}
\Lambda_{1}^{+} \\
\Lambda_{2}^{+} \\
\vdots \\
\Lambda_{N}^{+}
\end{array}\right]=\left[\begin{array}{c}
\Lambda_{1} \\
\Lambda_{2} \\
\vdots \\
\Lambda_{N}
\end{array}\right]+s\left[\begin{array}{c}
\Delta \Psi_{1}-U_{1}-a \Psi_{1}^{0} \\
\Delta \Psi_{2}-U_{2}+U_{1}-a \Psi_{2}^{0} \\
\vdots \\
\Delta \Psi_{N}-U_{N}+U_{N-1}-a \Psi_{N}^{0}
\end{array}\right]
$$

This demonstrates that every vehicle can also carry out Step 2 of the algorithm locally; vehicle 2 only needs to know $U_{1}$, vehicle 3 only needs to know $U_{2}$, and so on.

We observe that the dual subgradient algorithm facilitates local computation of the control signal at the expense of carrying out the computation iteratively. It is important to note that the solution thus obtained is in fact the global minimizer of the original optimal control problem.

The weakness of this method is that, once the iterations are complete, each vehicle will have computed its entire actuation profile and state trajectory from $k=0$ to $k=K-1$. The advantage is that each vehicle computes its actuation profile by communicating only with its immediate neighbors. This potentially makes the proposed method suitable for MPC implementation, where the time horizon $K$ can be taken to be relatively small (to minimize memory requirements at every vehicle), and the optimization procedure is repeated every $K$ time steps.

\section{Computation of Optimal Structured FEEDBACK GAINS}

In the previous section, we introduced a local algorithm in which each vehicle computed its optimal actuation profile and state trajectory. In what follows, however, we take a different approach by considering an a priori specified control structure that relates the state and actuation. This transforms the optimal control problem to a search for the optimal values of a set of structured control gains, rather than the search for the optimal values of actuation profiles.

We now revisit problem (LQR) of Section II, but with the additional requirement that for every time instant $k$ we have

$$
\begin{aligned}
u_{1}(k) & =f_{1} x_{1}(k), \\
u_{n}(k) & =f_{n}\left(x_{n}(k)-x_{n-1}(k)\right), \quad n=2, \cdots, N,
\end{aligned}
$$

where $f_{1}, \ldots, f_{N}$ are constant scalars. In other words, for all vehicles behind the lead vehicle, the control action of vehicle $n$ is proportional to its distance from vehicle $n-1$. Our objective is to find the optimal values of $f_{n}, n=1, \ldots, N$. We substitute this choice of $u_{n}$ into (LQR), and apply the change of variables

$$
\begin{aligned}
\psi_{1}(k) & :=x_{1}(k), \\
\psi_{n}(k) & :=x_{n}(k)-x_{n-1}(k), \quad n=2, \ldots, N .
\end{aligned}
$$


The optimal control problem now becomes

$$
\begin{aligned}
& \begin{array}{rr}
\operatorname{minimize} & \frac{1}{2} \sum_{k=0}^{K-1}\left(f_{1}^{2}+1\right) \psi_{1}^{2}+\cdots+\left(f_{N}^{2}+1\right) \psi_{N}^{2} \\
& +\frac{1}{2}\left[\psi_{1}^{2}(K)+\cdots+\psi_{N}^{2}(K)\right]
\end{array} \\
& \text { subject to } \psi_{1}(k+1)=\left(a+f_{1}\right) \psi_{1}(k) \text {, } \\
& \psi_{n}(k+1)=\left(a+f_{n}\right) \psi_{n}(k)-f_{n-1} \psi_{n-1}(k), \\
& n=2, \ldots, N \text {, } \\
& k=0, \ldots, K-1 \text {. }
\end{aligned}
$$

Using the vector representation $\Psi_{n}$, the optimization problem can be written as

$$
\begin{array}{cc}
\operatorname{minimize} & \frac{1}{2} \operatorname{tr}\left(\Psi_{1}^{T} Q_{1} \Psi_{1}\right)+\frac{1}{2}\left(f_{1}^{2}+1\right) \psi_{1}^{2}(0)+\cdots \\
& +\frac{1}{2} \operatorname{tr}\left(\Psi_{N}^{T} Q_{N} \Psi_{N}\right)+\frac{1}{2}\left(f_{N}^{2}+1\right) \psi_{N}^{2}(0)
\end{array}
$$

subject to $D_{1} \Psi_{1}+d_{1}+\cdots+D_{N} \Psi_{N}+d_{N}=0$

where

$$
\begin{aligned}
& Q_{n}\left(f_{n}\right)=\left[\begin{array}{cccc}
f_{n}^{2}+1 & & & \\
& \ddots & & \\
& & f_{n}^{2}+1 & \\
& & & 1
\end{array}\right], \quad n=1, \ldots, N, \\
& S=\left[\begin{array}{cccc}
0 & & & \\
1 & 0 & & \\
& \ddots & \ddots & \\
& & 1 & 0
\end{array}\right], D_{1}\left(f_{1}\right)=\left[\begin{array}{c}
\Delta-f_{1} S \\
f_{1} S \\
\vdots \\
0 \\
0
\end{array}\right], \cdots \\
& D_{N-1}\left(f_{N-1}\right)=\left[\begin{array}{c}
0 \\
0 \\
\vdots \\
\Delta-f_{N-1} S \\
f_{N-1} S
\end{array}\right], D_{N}\left(f_{N}\right)=\left[\begin{array}{c}
0 \\
0 \\
\vdots \\
0 \\
\Delta-f_{N} S
\end{array}\right] \text {, } \\
& d_{1}\left(f_{1}\right)=-\left[\begin{array}{c}
\left(a+f_{1}\right) \Psi_{1}^{0} \\
-f_{1} \Psi_{1}^{0} \\
\vdots \\
0 \\
0
\end{array}\right], \cdots \text {, } \\
& d_{N-1}\left(f_{N-1}\right)=-\left[\begin{array}{c}
0 \\
0 \\
\vdots \\
\left(a+f_{N-1}\right) \Psi_{N-1}^{0} \\
-f_{N-1} \Psi_{N-1}^{0}
\end{array}\right], d_{N}\left(f_{N}\right)=-\left[\begin{array}{c}
0 \\
0 \\
\vdots \\
0 \\
\left(a+f_{N}\right) \Psi_{N}^{0}
\end{array}\right]
\end{aligned}
$$

Forming the Lagrangian

$$
\begin{aligned}
L= & \frac{1}{2} \operatorname{tr}\left(\Psi_{1}^{T} Q_{1} \Psi_{1}\right)+\frac{1}{2}\left(f_{1}^{2}+1\right) \psi_{1}^{2}(0)+\cdots \\
& +\frac{1}{2} \operatorname{tr}\left(\Psi_{N}^{T} Q_{N} \Psi_{N}\right)+\frac{1}{2}\left(f_{N}^{2}+1\right) \psi_{N}^{2}(0) \\
& +\operatorname{tr}\left(\Lambda^{T}\left[D_{1} \Psi_{1}+d_{1}+\cdots+D_{N} \Psi_{N}+d_{N}\right]\right),
\end{aligned}
$$

the necessary conditions for optimality take the following form

$$
\begin{aligned}
& \frac{\partial L}{\partial f_{n}}=0 \Longrightarrow \\
& \left\|S \Psi_{n}+\Psi_{n}^{0}\right\|^{2} f_{n} \\
& -\operatorname{tr}\left(\left[\Lambda_{n}-\Lambda_{n+1}\right]^{T}\left[S \Psi_{n}+\Psi_{n}^{0}\right]\right)=0, \quad n=1, \ldots, N,
\end{aligned}
$$

(NEC1)

$$
\frac{\partial L}{\partial \Psi_{n}}=0 \Longrightarrow \Psi_{n}=-Q_{n}^{-1} D_{n}^{T} \Lambda, \quad n=1, \ldots, N
$$

$$
\frac{\partial L}{\partial \Lambda}=0 \Longrightarrow D_{1} \Psi_{1}+d_{1}+\cdots+D_{N} \Psi_{N}+d_{N}=0,
$$

(NEC3)

with $\Lambda_{N+1}=0$ in (NEC1).

Motivated by the development in the previous section, we propose the following algorithm.

\section{Dual subgradient algorithm for structured problem. repeat}

1. For given $\Lambda$, minimize Lagrangian in $f_{n}$ and $\Psi_{n}$ by simultaneously solving (NEC1) and (NEC2).

2. Update dual variables $\Lambda^{+}=\Lambda+s\left(D_{1} \Psi_{1}+d_{1}+\right.$ $\left.\cdots+D_{N} \Psi_{N}+d_{N}\right)$ for given step size $s$.

until stopping criterion $\| D_{1} \Psi_{1}+d_{1}+\cdots+D_{N} \Psi_{N}+$ $d_{N} \|<\varepsilon$ is satisfied.

It is easy to show that every vehicle can carry out the steps of this algorithm locally. To carry out Step 1, we have to simultaneously solve equations (NEC1) and (NEC2). By substituting the latter into the former, we obtain

$$
\begin{aligned}
& 0=\psi_{n}^{2}(0) f_{n}+\left\|S \Delta^{T} \Lambda_{n}\right\|^{2} \frac{f_{n}}{\left(f_{n}^{2}+1\right)^{2}} \\
& -2 \operatorname{tr}\left(\left[\Lambda_{n}-\Lambda_{n+1}\right]^{T} S S^{T} S \Delta^{T} \Lambda_{n}\right) \frac{f_{n}^{2}}{\left(f_{n}^{2}+1\right)^{2}} \\
& +\left\|S S^{T}\left[\Lambda_{n}-\Lambda_{n+1}\right]\right\|^{2} \frac{f_{n}^{3}}{\left(f_{n}^{2}+1\right)^{2}}-\left\|S^{T}\left[\Lambda_{n}-\Lambda_{n+1}\right]\right\|^{2} \frac{f_{n}}{f_{n}^{2}+1} \\
& +\operatorname{tr}\left(\left[\Lambda_{n}-\Lambda_{n+1}\right]^{T} S \Delta^{T} \Lambda_{n}\right) \frac{1}{f_{n}^{2}+1}-\operatorname{tr}\left(\left[\Lambda_{n}-\Lambda_{n+1}\right]^{T} \Psi_{n}^{0}\right)
\end{aligned}
$$

for $n=1, \ldots, N$, with $\Lambda_{N+1}=0$. If we multiply through by $\left(f_{n}^{2}+1\right)^{2}$, we arrive at fifth-order polynomial equations (in the unknown $f_{n}$ 's) whose solutions identify all the extremum points of $L$. In the case of multiple solutions, we choose the value of $f_{n}$ that minimizes the Lagrangian. It is noteworthy that the order of these equations is independent of the number of vehicles.

Remark 2: Vehicle $n$ chooses $f_{n}$ so as to minimize

$$
\begin{aligned}
L_{n}=\frac{1}{2} \operatorname{tr}\left(\Psi_{n}^{T} Q_{n} \Psi_{n}\right) & +\frac{1}{2}\left(f_{n}^{2}+1\right) \psi_{n}^{2}(0) \\
& +\operatorname{tr}\left(\Lambda^{T}\left[D_{n} \Psi_{n}+d_{n}\right]\right),
\end{aligned}
$$

where $L_{n}$ is the part of the Lagrangian that corresponds to the $n$th vehicle, and $L=L_{1}+\cdots+L_{N}$. Note that vehicle $n$ can choose its feedback gain $f_{n}$ independently of the other vehicles. In particular, given $\Lambda$, if the fifth-order equation at vehicle $n$ has multiple solutions then $f_{n}$ is chosen so as to minimize $L_{n}$.

Once each $f_{n}$ is found then $Q_{n}$ and $D_{n}$ are known and thus $\Psi_{n}$ is obtained from (NEC2). Furthermore, from (NEC2) it follows that for $n=1, \ldots, N$,

$$
\begin{aligned}
\Psi_{n} & =-Q_{n}^{-1} D_{n}^{T} \Lambda \\
& =-Q_{n}^{-1}\left(\Delta^{T}-f_{n} S^{T}\right) \Lambda_{n}-Q_{n}^{-1} f_{n} S^{T} \Lambda_{n+1},
\end{aligned}
$$

with $\Lambda_{N+1}=0$, and thus $\Psi_{n}$ can be obtained locally. Finally, 
Step 2 of the algorithm gives

$$
\begin{aligned}
& {\left[\begin{array}{c}
\Lambda_{1}^{+} \\
\Lambda_{2}^{+} \\
\vdots \\
\Lambda_{N}^{+}
\end{array}\right]=\left[\begin{array}{c}
\Lambda_{1} \\
\Lambda_{2} \\
\vdots \\
\Lambda_{N}
\end{array}\right]} \\
& +s\left[\begin{array}{c}
\left(\Delta-f_{1} S\right) \Psi_{1}-\left(a+f_{1}\right) \Psi_{1}^{0} \\
\left(\Delta-f_{2} S\right) \Psi_{2}+f_{1} S \Psi_{1}-\left(a+f_{2}\right) \Psi_{2}^{0}+f_{1} \Psi_{1}^{0} \\
\vdots \\
\left(\Delta-f_{N} S\right) \Psi_{N}+f_{N-1} S \Psi_{N-1}-\left(a+f_{N}\right) \Psi_{N}^{0}+f_{N-1} \Psi_{N-1}^{0}
\end{array}\right] .
\end{aligned}
$$

It is important to note here that the above update expression shows not only that the dual variables can be updated locally, but also that each vehicle can independently determine whether it has reached convergence; each vehicle can decide to terminate its iterations if its respective term inside the last vector of the equation above is small enough.

\section{Relaxation of StRuctured PRoblem}

Problem (SH2) is a nonconvex optimization problem with possibly many local minima, which manifests itself in equations (5) possibly having multiple solutions for $f_{n}$. This may prevent convergence of the dual subgradient algorithm of the previous section. The augmented Lagrangian method [19], [21] provides a way of locally convexifying an optimization problem around its constraint set. This is achieved by adding a quadratic form of the constraints to the Lagrangian. For example, the (standard) augmented Lagrangian for the problem

$$
\begin{array}{ll}
\text { minimize } & J(Z) \\
\text { subject to } & H(Z)=0
\end{array}
$$

is given by

$$
L_{a}=J+\operatorname{tr}\left(\Lambda^{T} H\right)+\frac{\sigma}{2} \operatorname{tr}\left(H^{T} H\right),
$$

where $\Lambda$ is the dual variable and $\sigma$ is a positive scalar. We will refer to $\frac{\sigma}{2} \operatorname{tr}\left(H^{T} H\right)$ as the penalty term.

The augmented Lagrangian method is most effective when $H$ is a convex function of the optimization variable $Z$. However, this is not the case in problem $(\mathrm{SH} 2)$, since $\Psi_{n}$ and $f_{n}$ are both optimization variables and each $D_{n}$ is a function of $f_{n}$. Moreover, it can be shown that the addition of $\operatorname{tr}\left(H^{T} H\right)$ to the Lagrangian of problem ( $\mathrm{SH} 2$ ) will destroy the possibility of localized computations. We therefore propose a relaxed augmented Lagrangian for problem (SH2).

Let

$$
\begin{aligned}
H_{1}\left(f_{1}\right):= & D_{1}\left(f_{1}\right) \widetilde{\Psi}_{1}+d_{1}\left(f_{1}\right)+D_{2}\left(\widetilde{f}_{2}\right) \widetilde{\Psi}_{2}+d_{2}\left(\widetilde{f}_{2}\right) \\
& +\cdots+D_{N}\left(\widetilde{f}_{N}\right) \widetilde{\Psi}_{N}+d_{N}\left(\widetilde{f}_{N}\right), \\
\vdots & \\
H_{N}\left(f_{N}\right):= & D_{1}\left(\widetilde{f}_{1}\right) \widetilde{\Psi}_{1}+d_{1}\left(\widetilde{f}_{1}\right)+D_{2}\left(\widetilde{f}_{2}\right) \widetilde{\Psi}_{2}+d_{2}\left(\widetilde{f}_{2}\right) \\
& +\cdots+D_{N}\left(f_{N}\right) \widetilde{\Psi}_{N}+d_{N}\left(f_{N}\right),
\end{aligned}
$$

where tilded parameters are assumed known and are not optimization variables. We later choose the value of the tilded parameters to be equal to the value of their untilded counterparts from the previous iteration; we will justify the rationale for such a choice after we present the main algorithm. We also emphasize that each $H_{n}$ is affine in the variable $f_{n}$.

The relaxed augmented Lagrangian is now defined as

$$
\begin{aligned}
L_{a}= & \frac{1}{2} \operatorname{tr}\left(\Psi_{1}^{T} Q_{1} \Psi_{1}\right)+\frac{1}{2}\left(f_{1}^{2}+1\right) \psi_{1}^{2}(0)+\cdots \\
& +\frac{1}{2} \operatorname{tr}\left(\Psi_{N}^{T} Q_{N} \Psi_{N}\right)+\frac{1}{2}\left(f_{N}^{2}+1\right) \psi_{N}^{2}(0) \\
& +\operatorname{tr}\left(\Lambda^{T}\left[D_{1} \Psi_{1}+d_{1}+\cdots+D_{N} \Psi_{N}+d_{N}\right]\right) \\
& +\frac{\sigma}{2}\left[\operatorname{tr}\left(H_{1}^{T} H_{1}\right)+\cdots+\operatorname{tr}\left(H_{N}^{T} H_{N}\right)\right] .
\end{aligned}
$$

Setting

$$
\frac{\partial L_{a}}{\partial \Psi_{n}}=0 \quad \text { and } \quad \frac{\partial L_{a}}{\partial \Lambda}=0
$$

leads to the same equations as in (NEC2)-(NEC3). Setting

$$
\frac{\partial L_{a}}{\partial f_{n}}=0
$$

(CVX)

again leads to fifth-order equations as in (5). However, this time the coefficients are functions of $\sigma$. Furthermore, it is observed that if $\widetilde{f}_{n}$ and $\widetilde{\Psi}_{n}$ are chosen appropriately then for large enough values of $\sigma$ these polynomial equations always have a unique solution for $f_{n}$. For brevity, we omit the exact expressions for these coefficients; they will be reported elsewhere.

We thus propose the following algorithm, which also describes the procedure for choosing the tilded parameters.

Dual subgradient algorithm for relaxed problem.

given $\widetilde{\Psi}_{n}=0, \widetilde{f}_{n}=0$, and $\sigma>0$.

repeat

1. For given $\Lambda, \widetilde{\Psi}_{n}, \widetilde{f}_{n}$, and $\sigma$, solve the polynomial equations in $f_{n}$ resulting from (CVX).

2. Compute $\Psi_{n}$ from (NEC2).

3. Update dual variables $\Lambda^{+}=\Lambda+s\left(D_{1} \Psi_{1}+d_{1}+\right.$ $\left.\cdots+D_{N} \Psi_{N}+d_{N}\right)$ for given step size $s$.

4. Set $\widetilde{\Psi}_{n}=\Psi_{n}, \widetilde{f}_{n}=f_{n}$, and $\sigma^{+}=\tau \sigma$ for some $\tau>1$.

until stopping criterion $\| D_{1} \Psi_{1}+d_{1}+\cdots+D_{N} \Psi_{N}+$ $d_{N} \|<\varepsilon$ is satisfied.

Again, all steps of this algorithm can be carried out locally. This is particularly true for the polynomial equations that follow from $(\mathrm{CVX})$; it can be shown that the coefficients of the fifth-order polynomial in $f_{n}$ depend only on $\Lambda_{n}, \Lambda_{n+1}, \widetilde{\Psi}_{n-1}, \widetilde{\Psi}_{n}, \widetilde{\Psi}_{n+1}, \widetilde{f}_{n-1}$, and $\widetilde{f}_{n+1}$. When convergence is achieved, the values of $\Psi_{n}$ and $f_{n}$ stay constant over different iterations. Thus we have $H_{1}^{T} H_{1}=\cdots=H_{N}^{T} H_{N}=H^{T} H$, and the relaxed augmented Lagrangian (AL) converges to the standard augmented Lagrangian (modulo a rescaling of the penalty coefficient $\sigma$ ).

Finally, we note that when the algorithm in Section V converges, its solution agrees with the solution of the convexfied problem in this section. However, the algorithm in Section V does not always converge whereas the algorithm in this section always does. These observations are expected, as the augmented Lagrangian method does not alter the solution of problems whose Lagrangian already has a unique minimum. 


\section{NUMERICAL EXAMPLES}

We consider an optimal control problem over a time horizon of $K=10$ for a system with $N=4$ vehicles. A step size of $s=0.001$ is used throughout. The first set of plots shown in Fig. 1 correspond to a system with $a=-0.8$, and the second set of plots shown in Fig. 2 correspond to a system with $a=1$.
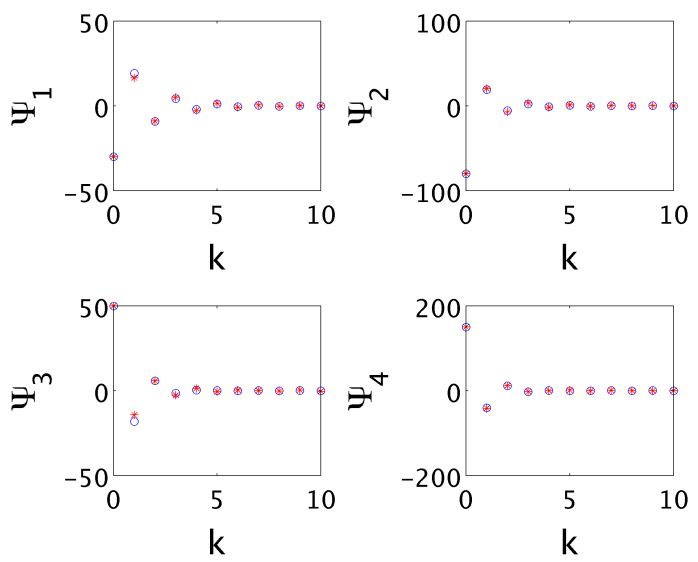

Fig. 1. The trajectory of every vehicle in a system with 4 vehicles and $a=-0.8$. Blue circles correspond to $(\mathrm{H} 2)$ and red stars correspond to (SH2). The difference between the performance of the (H2) and (SH2) problems is $0.17 \%$.
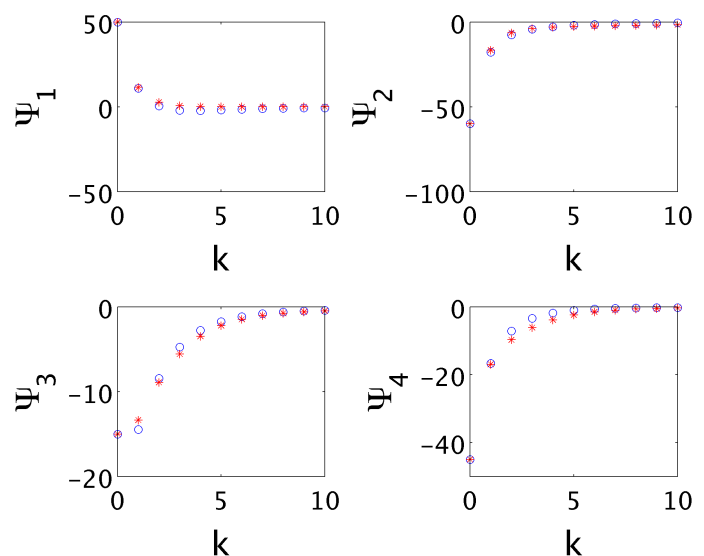

Fig. 2. The trajectory of every vehicle in a system with 4 vehicles and $a=+1.0$. Blue circles correspond to (H2) and red stars correspond to (SH2). The difference between the performance of the (H2) and (SH2) problems is $0.76 \%$.

\section{CONCLUSIONS}

We use the dual decomposition method to decouple the optimal control problem for vehicular formations. This produces optimal control laws in a localized manner, in the sense that vehicles iteratively compute their primal and dual variables by only communicating with their immediate neighbors. We then develop an algorithm by which vehicles can compute structured feedback gains in a localized manner. This is in the same spirit as [22] where, for the LQR problem, feedback gains are sought with an $a$ priori specified localized structure. To improve numerical properties of the dual subgradient method we use a relaxed version of the augmented Lagrangian method, which renders a locally convex problem and behaves like the standard augmented Lagrangian when convergence is achieved.

Future work in this direction would include reformulating the structured control problem in a stochastic programming [18] setting, so as to obtain feedback gains that do not depend on the initial conditions of the vehicles.

\section{REFERENCES}

[1] B. Bamieh, F. Paganini, and M. A. Dahleh, "Distributed control of spatially invariant systems," IEEE Transactions on Automatic Control, vol. 47, no. 7, pp. 1091-1107, 2002.

[2] G. A. de Castro and F. Paganini, "Convex synthesis of localized controllers for spatially invariant system," Automatica, vol. 38, pp. 445-456, 2002.

[3] P. G. Voulgaris, G. Bianchini, and B. Bamieh, "Optimal $H^{2}$ controllers for spatially invariant systems with delayed communication requirements," Systems and Control Letters, vol. 50, pp. 347-361, 2003.

[4] R. D'Andrea and G. E. Dullerud, "Distributed control design for spatially interconnected systems," IEEE Transactions on Automatic Control, vol. 48, no. 9, pp. 1478-1495, 2003.

[5] G. Dullerud and R. D'Andrea, "Distributed control of heterogeneous systems," IEEE Transactions on Automatic Control, vol. 49, no. 12 , pp. 2113-2128, December 2004.

[6] C. Langbort, R. S. Chandra, and R. D'Andrea, "Distributed control design for systems interconnected over an arbitrary graph," IEEE Transactions on Automatic Control, vol. 49, no. 9, pp. 1502-1519, September 2004.

[7] B. Bamieh and P. G. Voulgaris, "A convex characterization of distributed control problems in spatially invariant systems with communication constraints," Systems and Control Letters, vol. 54, pp. 575-583, 2005.

[8] M. Rotkowitz and S. Lall, "A characterization of convex problems in decentralized control," IEEE Transactions on Automatic Control, vol. 50, no. 12, pp. 1984-1996, 2005.

[9] A. Rantzer, "Linear quadratic team theory revisited," in Proceedings of the 2006 American Control Conference, 2006, pp. 1637-1641.

[10] - "A separation principle for distributed control," in Proceedings of the 45th IEEE Conference on Decision and Control, 2006, pp. 36093613.

[11] N. Motee and A. Jadbabaie, "Optimal control of spatially distributed systems," IEEE Transactions on Automatic Control, vol. 53, no. 7, pp. 1616-1629, Aug. 2008.

[12] F. Borrelli and T. Keviczky, "Distributed LQR design for identical dynamically decoupled systems," IEEE Transactions on Automatic Control, vol. 53, no. 8, pp. 1901-1912, Sept. 2008.

[13] M. Fardad and M. R. Jovanović, "On the state-space design of optimal controllers for distributed systems with finite communication speed," in Proceedings of the 47th IEEE Conference on Decision and Control, 2008, pp. 5488-5493.

[14] K. Mårtensson and A. Rantzer, "Gradient methods for iterative distributed control synthesis," in Proceedings of the 48th IEEE Conference on Decision and Control, 2009, pp. 549-554.

[15] A. Rantzer, "Dynamic dual decomposition for distributed control," in Proceedings of the 2009 American Control Conference, 2009, pp. 884 888.

[16] R. L. Raffard, C. J. Tomlin, and S. Boyd, "Distributed optimization for cooperative agents: Application to formation flight," in Proceedings of the 43rd IEEE Conference on Decision and Control, 2004, pp. 24532459.

[17] I. Necoara and J. A. K. Suykens, "A proximal center-based decomposition method for multi-agent convex optimization," in Proceedings of the 47th IEEE Conference on Decision and Control, 2008, pp. 30773082.

[18] S. Boyd, Notes for EE364b: Convex Optimization II, Stanford University, 2008.

[19] D. G. Luenberger and Y. Ye, Linear and Nonlinear Programming. Springer, 2008.

[20] D. P. Bertsekas and J. N. Tsitsiklis, Parallel and Distributed Computation: Numerical Methods. Athena Scientific, 1997.

[21] D. P. Bertsekas, Nonlinear Programming. Athena Scientific, 2008.

[22] M. Fardad, F. Lin, and M. R. Jovanović, "On the optimal design of structured feedback gains for interconnected systems," in Proceedings of the 48th IEEE Conference on Decision and Control, 2009, pp. 978983. 